\title{
Espacios de trabajo para profesionales de la información en Brasil: resultados preliminares
}

\author{
Miriam Vieira da Cunha \\ Universidade Federal de Santa Catarina (Brasil)
}

\section{Resumen}

El mundo actual puede comprenderse a través de sus múltiples facetas culturales, políticas, económicas y sociales. Los cambios en la sociedad del conocimiento están llevando a los profesionales de la información a redefinir sus roles en los espacios tradicionales de actuación. Estos nuevos aspectos del trabajo crean nuevas relaciones entre las profesiones y posibilitan, en algunos casos, la ruptura de las fronteras entre ellas. La explosión de la comunicación a través de Internet y la adición del valor de la información como recurso estratégico llevan cada vez a más personas a trabajar con fuentes de información. Este movimiento crea tensiones y nuevas oportunidades de alianzas en este espacio. La combinación de estos movimientos, tensiones y alianzas forma un campo de competencia nuevo o, mejor dicho, una nueva jurisdicción profesional (Abbott, 1988). Las listas de discusión de ciencias de la información y sitios específicos divulgan en Brasil, desde el año 2000, ofertas de trabajo para profesionales de la información. El objetivo de este estudio es conocer y caracterizar las ofertas de empleo para profesionales de la información disponibles en Internet entre 2005 y 2006. Utiliza la técnica de análisis de contenido de Bardin (2004) y tiene como criterios de análisis la fecha del anuncio, la fuente de información, el tipo de profesional, la ciudad y la formación y función solicitadas. Los primeros resultados evidencian que la mayoría de las ofertas son para bibliotecarios y archiveros y se concentran en las grandes ciudades brasileñas, como Río de Janeiro y São Paulo.

Palabras clave: Profesionales de la información. Perfil profesional. Brasil.

\begin{abstract}
The world today can be understood through its multiple cultural, political, economical and social spheres. Transformations in the information community are leading information professionals to redefine their roles in their traditional work areas. These new aspects of work are creating new relationships between professions and, in some cases, are breaking down barriers between them. The explosion of the Internet communication and the increase in value of information
\end{abstract}


as a strategic resource are leading growing numbers of people to work with information sources. This movement is creating tensions as well as new opportunities for partnerships in the field. The combination of these movements, tensions and partnerships have formed a new field of competence, or rather, a new professional jurisdicition (Abbott, 1988). Discussion lists for Brazilian Information Sciences and sites focussing on the field have, since 2000, published job vacancies for information professionals. With this in mind, this study aims to discover and define job vacancies for Brazilian information professionals available on the Internet between 2005 and 2006. It uses Bardin's (2004) technique of content analysis, and uses the following analysis criteria: subject date, information source, profession type, city, qualifications and required functions. The first results have shown that most vacancies are for librarians and archivists and are centred around major Brazilian cities, such as Rio de Janeiro and São Paulo.

Key words: Information professionals. Professional profile. Brazil.

\section{Introducción}

La realidad de hoy en día, caracterizada por el uso intensivo de la información, puede comprenderse a través de sus diversas facetas culturales, políticas, económicas y sociales. Estas múltiples realidades modifican el conocimiento y el quehacer humanos. Sin duda, el desarrollo y la difusión del nuevo paradigma tecnológico, impulsado por la vinculación de las tecnologías electrónicas, ópticas, de multimedia y de comunicación, constituyen la base que sostiene estos cambios y llevan al cuestionamiento de muchos paradigmas y a una explosión de la comunicación sin precedentes en la historia de la humanidad.

Estas transformaciones son parte del proceso de fragmentación y dispersión del mundo del trabajo (Moore, 1999). Se crean nuevas asociaciones que constituyen una nueva delimitación de los espacios profesionales. Como consecuencia de estos cambios, el sistema de las profesiones y sus articulaciones pasan en este momento por una reorganización. Estas transformaciones permiten que surjan nuevas ocupaciones al mismo tiempo que se destruyen otras. En efecto, "las tecnologías abren una gama extremadamente diversificada de áreas de trabajo, lo que confunde nuestras visiones del trabajo y de la profesión”. (Dowbor, 2001, pp. 17-18)

Todo progreso, según Cronin (1998), trae consigo un fenómeno de destrucción. El proceso de integración de una nueva tecnología perturba en un primer momento el funcionamiento de la economía, y enseguida exige una adaptación de las estructuras y de los comportamientos.

Los cambios tecnológicos alteran de forma drástica las prácticas profesionales en todas las áreas del conocimiento. Esto repercute notablemente en el mundo laboral, que se está reorganizando, lo que aporta inseguridad a los profesionales. 
Este proceso de transformación profesional es inherente al nuevo modelo económico, que introduce nuevas formas de gestión del trabajo y de socialización, valorando las actividades en equipo, la interdisciplinaridad y el aprendizaje continuo. Sin embargo, esta "subversión" de espacios es más aguda en el campo de las profesiones de la información. Más aguda porque los límites que históricamente demarcaban los diferentes tipos de trabajo en el ámbito de la información se han atenuado. Esta revolución en el mundo laboral lleva a cambios intensos, a nuevas formas de intermediación o a reintermediaciones (Cronin, 1998).

Estas tecnologías provocan la convergencia de áreas que antes, cuando existía la posibilidad de reconocer "campos de trabajo" privativos de una categoría profesional, eran estancas. En realidad, al dominio de las actividades de la información se han agregado nuevos profesionales en contextos cada vez más dinámicos. El contexto de la Web, por ejemplo, como resaltan García Marco y Agustín (2000), "disolvió" la frontera entre la producción y la comunicación de la información.

Tales evoluciones parecen caminar hacia la organización de espacios donde profesionales de distintos orígenes trabajen de forma interactiva, creando nuevas relaciones profesionales y llegando, incluso, a la ruptura de las fronteras establecidas.

Hemos afirmado anteriormente (Cunha, 2002; Cunha y Crivellari, 2006) que el sector de la información es, por su propia naturaleza, un sector heterogéneo. Se trata además de un espacio interdisciplinario por excelencia (Saracevic, 1995) (1), ocupado por profesionales originarios de diferentes áreas del conocimiento. Tal como ya hemos señalado,

la diversidad de los ambientes profesionales donde se realizan estas actividades, así como la de sus actores, y la interpenetración relativa de sus funciones hacen realmente difícil la caracterización de los espacios de información. En este sentido, los límites que separan las ocupaciones de este sector no están claros. En realidad, la evolución de la sociedad de la información da la impresión de una confusión de pistas, una yuxtaposición de espacios, de actores y de funciones. (Cunha y Crivellari, 2006, p. 19)

Para Mueller,

el uso de los términos profesiones de la información y profesionales de la información es habitual en las últimas décadas en la literatura, y refleja la comprensión de que, hoy en día, los servicios de información presentan una gran complejidad y demandan más que el trabajo aislado de una profesión. Entre tanto, el significado exacto de los términos no está claro en relación con los profesionales, trabajos o servicios a los que se refieren. Ciertamente, existe el consenso de que hay ciertas características mínimas comunes a todos los profesionales de la información, lo que permite el uso de este término en varios contextos. Pero la comprensión parece depender de quién utiliza el término y de la audiencia a la que se dirige. En general, parece haber consenso en que entre los profesionales de la información están los bibliotecarios, los archiveros y los que tienen el posgrado y el doctorado en Ciencia de la Información. (Mueller, 2004, p. 23)

Scire. 12 : 2 (jul.-dic. 2006) 27-36. ISSN 1135-3716. 
Las tareas relacionadas con el trabajo informacional se hacen más complejas y específicas. Esta tendencia a la especialidad no ha resultado en el fortalecimiento de las profesiones "clásicas" de la información (bibliotecarios, archiveros, documentalistas y museólogos), pero ha ofrecido posibilidades a especialistas de otras áreas del conocimiento. De esta forma, el desarrollo de la industria de la información ha abierto, efectivamente, este campo a una amplia población de profesionales de varias áreas del conocimiento, que trabajan de un modo interactivo en distintos espacios. El trabajo informacional es, cada vez más, un trabajo de equipo - con profesionales de distintas formaciones-. Estos nuevos espacios de trabajo interdisciplinario crean nuevas relaciones entre las profesiones, posibilitando, en algunos casos, la ruptura de las fronteras y de los límites profesionales anteriormente establecidos.

Las transformaciones que se producen en las profesiones relacionadas con la información reflejan la evolución del sistema de las profesiones (a partir de la teoría de Abbott, 1998) y apuntan, como afirman Cronin, Stiffler y Day (1993), a cambios desde un núcleo tradicional, conocido y delimitado, hacia una periferia. Estos autores afirman, partiendo de la lectura de Abbott, que los cambios profesionales tienen lugar siempre al borde de este núcleo conocido y bien delimitado. En lo que se refiere a las profesiones de la información, estas transformaciones son el resultado de la diversificación de los soportes, de las funciones, de los papeles, de los usos y de las expectativas de los usuarios en relación con la disponibilidad de la información.

Los profesionales de la información son llevados, cada vez más, a participar activamente del flujo internacional de informaciones a través de la oferta de trabajo a usuarios virtuales que pueden estar en cualquier lugar del planeta. En contrapartida, estos mismos profesionales se benefician y utilizan servicios procedentes de ese flujo internacional. Su trabajo es, esencialmente, una labor de difusión de información desde un contexto local —el de la institución y el de la unidad de información donde trabajan - a un contexto planetario, y de este al individual.

La explosión de la comunicación a través de Internet y el aumento del valor de la información como recurso estratégico llevan cada vez a más individuos a trabajar con información. Este movimiento crea tensiones y nuevas oportunidades de alianzas en este espacio. La combinación de estos movimientos, tensiones y alianzas forma un campo de competencia nuevo, o una nueva jurisdicción profesional (2), en el sentido que Abbott (1998) da a este término. Esta nueva jurisdicción nace de la confluencia e interacción de varios campos profesionales en un intersticio de interprofesionalización, un área de actuación donde se encuentran informáticos, bibliotecarios, gestores y periodistas, entre otros.

En este contexto de transformaciones, de necesidades complejas y diversificadas, Internet se presenta como una pantalla rica, pero fragmentada, de infor-

Scire. 12 : 2 (jul.-dic. 2006) 27-36. ISSN 1135-3716. 
maciones que crecen de un modo exponencial. La Red ha provocado cambios sustanciales que tienen un impacto extraordinario en todos los sectores de la vida: el político, el económico, el cultural, el educativo y el laboral. En los últimos años están surgiendo profesionales preocupados por "dar sentido" a estas transformaciones, por evaluar y recuperar los contenidos de la Red.

Internet, además de todas sus funciones o metafunciones, por utilizar el concepto de Cronin (1998), sirve cada vez más para la divulgación de ofertas de trabajo, de concursos y, en fin, de nuevas oportunidades para el profesional de la información.

Todo hace pensar que el uso intensivo de las tecnologías ha facilitado y simplificado los procesos de transmisión de la información. En el caso de las ofertas de trabajo, nos parece evidente que Internet está facilitando esta propagación al reducir el tiempo que transcurre entre la generación de la oferta y su difusión (Arévalo y Vázquez, 2000).

En Brasil, la divulgación de ofertas para profesionales de la información es dispersa y reducida. La investigación de Cunha (1998) apunta a los periódicos — por ejemplo, O Globo, Folha de São Paulo, Jornal do Brasil y Estado de São Paulocomo los instrumentos que más las difunden. Sin embargo, el bajo número de ofertas publicadas en estos periódicos nos lleva a creer que este tipo de anuncios se propagan de otras formas, como, por ejemplo, a través de Internet.

El objetivo de este estudio ha sido conocer y caracterizar las ofertas de empleo para profesionales de la información disponibles en Internet y difundidas en sitios específicos y listas de discusión entre 2005 y 2006. Se ha utilizado la técnica de análisis de contenido de Bardin (2004) y los criterios de análisis han sido la fecha del anuncio, la fuente de información, el tipo de profesional, la ciudad y la formación, experiencia profesional y función solicitadas.

\section{Análisis de los datos}

Aunque sea cada vez más evidente el papel que la información desempeña en la sociedad actual, ocasionando modificaciones importantes en el campo de las actividades relacionadas con la información, en Brasil la evolución de esta área y de sus profesionales es aún muy limitada, según se puede verificar con los datos que se presentan a continuación.

Entre enero de 2005 y junio de 2006 se divulgaron en los sitios y listas de discusión analizados 1076 anuncios para profesionales de la información. La gran mayoría (o sea, el 76,26\%) solicitaba bibliotecarios; luego estaban las ofertas para archiveros (que representan el 9,6\%), para auxiliares de biblioteca (el 4,5\%) y para gestores de información (el 3,7\%). La demanda de auxiliares de biblioteca se justifica, a nuestro entender, por dos motivos: $a$ ) la "confusión de límites entre el

Scire. 12 : 2 (jul.-dic. 2006) 27-36. ISSN 1135-3716. 
trabajo del profesional bibliotecario y de su auxiliar" (Danner, 1998); b) razones económicas: si un auxiliar puede ejecutar el trabajo de un profesional, es más barato contratar a un auxiliar.

Lo que se deduce de estos datos es que, en Brasil, el profesional solicitado para la mayoría de las unidades de información continúa siendo el titulado en Biblioteconomía. En realidad, a pesar de que las universidades brasileñas empiezan a presentar una diversidad de titulaciones en el área de estudios de información, con cursos de Gestión de la Información, Sistemas de Información, Ciencia de la Información y Administración de Información, entre otros, los estudios de Biblioteconomía continúan siendo los más "visibles", con 35 escuelas en el país. Los cursos de Archivística, por ejemplo, suman apenas 7, los de Gestión de la Información, 3, y los de Museología, 2 (ABECIN, 2006).

Una de las razones de esta concentración de demandas de bibliotecarios está originada, a nuestro entender, por la legislación profesional brasileña. Hay que señalar que en Brasil, para trabajar en una biblioteca, el profesional debe haber hecho un curso de graduado en Biblioteconomía. Esta formación es la única que permite la inscripción en uno de los Consejos de Biblioteconomía, instituciones que reglamentan la profesión en Brasil (Brasil. Decreto 56726, 1965) (3). Esta reglamentación, hasta el momento, ha sido utilizada para intentar impedir el ejercicio legal de la profesión a quienes tienen un diploma de maestría o un doctorado en Ciencia de la Información, y una formación inicial en otra área del conocimiento.

Además, en relación con la formación en Gestión de la Información, un área relativamente nueva en Brasil, los Consejos de Biblioteconomía prohíben a los profesionales titulados por estas escuelas trabajar en bibliotecas, espacios solo abiertos legalmente a los bibliotecarios. Se trata, a nuestro entender, de una situación absurda que no debería mantenerse.

Las ofertas de empleo analizadas demuestran que las profesiones de la información más buscadas continúan siendo las clásicas "tres marías", en palabras de Johanna Smit (1994), o sea, bibliotecarios, archiveros y museólogos. Hay que mencionar la aparición de demandas de museólogos, algo bastante raro en las ofertas de empleo brasileñas, en los últimos diez años. Nos parece importante subrayar que

tanto el bibliotecario como el archivero y el museólogo son miembros de una misma familia, la familia de los profesionales de la información, que actúan, a pesar de especialidades indiscutibles, con objetivos comunes. (Smit, 1994)

Por otra parte, cerca del $15 \%$ de los anuncios solicitan, en una misma oferta, bibliotecarios o archiveros indistintamente; en el 3\% de los casos se pide en un mismo anuncio un bibliotecario, un archivero o museólogo. A título de comparación, en las ofertas brasileñas analizadas por Cunha en 1998, el 10,7\% solicitaban, indistintamente, un archivero o un bibliotecario. Este tipo de oferta puede tener, en nuestra

Scire. 12 : 2 (jul.-dic. 2006) 27-36. ISSN 1135-3716. 
opinión, dos significados: a) el empleador desconoce el área y por eso "se arriesga"; b) el empleador no sabe qué profesional quiere, así que solicita todos. Este tipo de reflexión ya había sido hecho por Cunha (1998) durante el análisis de las ofertas para profesionales de la información publicadas en anuncios franceses y brasileños.

En lo que se refiere a la formación, el $24 \%$ solicitan un profesional con titulación superior, sin decir cuál; el 15,4\% piden formación en Biblioteconomía; el $1 \%$, en Archivística, y el 0,5\%, en Archivística o Biblioteconomía. Todo hace pensar que, aunque se demande, en la mayoría de los casos, un bibliotecario, el empleador no relaciona a este profesional con su formación específica, lo que quizás implica un desconocimiento de la misma.

En relación con el conocimiento de idiomas, apenas el 6,53\% de las ofertas solicita esta competencia. De ellas, la inmensa mayoría demanda el inglés. Todo parece indicar que la mayoría de los empleadores consideran que los profesionales saben idiomas, y que no es necesario explicitar esta información en las ofertas. En el mundo actual, de globalización y dominio de las redes en la búsqueda y análisis de la información, el conocimiento de idiomas es fundamental, como subrayan Tomael y Alvarenga (2000), Marchiori (1996) y Montalli (1997), entre otros.

En cuanto a la experiencia profesional, cerca del $25 \%$ de las ofertas analizadas solicitan esta información. La mayoría pide experiencia en informática, fundamental para los profesionales de la información hoy en día.

São Paulo aparece como la ciudad que más profesionales de la información solicita en los últimos años, con el 25\% de las demandas. Esto no es una sorpresa, ya que esta ciudad es la mayor y más importante ciudad brasileña, además de tener el mayor producto interior bruto del país. En este sentido, es lógico que concentre el mayor número de ofertas de empleo. A continuación aparece Río de Janeiro con el 18\%; Brasilia tiene el 5\%.

\section{Conclusión}

Hay que destacar que esta encuesta es una fotografía de una realidad en un momento preciso y en un ambiente en permanente evolución. En cuanto a la pluralidad de profesionales solicitados en cerca del 15\% de las ofertas, las encuestas de Cronin, Stiffler y Day (1993), Chen, Raskin y Tebbets (1984) y Moore (1987) mencionan la falta de consenso de los empleadores con respecto al perfil del profesional que buscan. Además, estos resultados tienden a demostrar la falta de distinción existente entre las diferentes profesiones de la información, especialmente entre las "tres marías" - bibliotecarios, archiveros y museólogos (Danner, 1998).

Por otra parte, esta encuesta corrobora resultados de encuestas anteriores, como la de Tarapanoff (1997), al destacar la demanda de bibliotecarios en las unidades de información. 
En realidad, a pesar de las transformaciones, de la reorganización del espacio de trabajo y de las nuevas colaboraciones interprofesionales que se crearon en el campo informacional en Brasil, este campo aún muestra resistencia a la apertura, con pocas alteraciones. Podemos concluir que las "oportunidades de mercado" en el área de la información continúan dominadas por los profesionales "clásicos", o sea, bibliotecarios, archiveros y museólogos. Los datos demuestran que el profesional tipo solicitado a través de sitios específicos y listas de discusión en Internet entre enero de 2005 y junio de 2006 es $a$ ) un bibliotecario, $b$ ) con formación superior, $c$ ) con experiencia en informática, $d$ ) para trabajar en la ciudad de São Paulo.

Lo que se hace evidente, según el estudio, es que, a pesar de que dan prioridad a un profesional "clásico", los empleadores no distinguen, en muchos casos, el tipo de profesional que quieren. Esta falta de diferenciación es, a nuestro entender, resultado de las transformaciones en los espacios de trabajo. Para concluir, se puede afirmar que, aunque estemos viviendo un momento de transformación en el mundo laboral, en Brasil el espacio de los profesionales de la información aún es percibido como un espacio para los profesionales tradicionales. En este sentido, el conflicto y la lucha por la jurisdicción en esta área son todavía incipientes en nuestro país.

\section{Notas}

(1) La cuestión de la comprensión de la información, de su comunicación, de sus manifestaciones y de sus efectos en el comportamiento de los usuarios, así como el problema de su accesibilidad, no pueden ser resueltos por una única área del conocimiento. La interdisciplinaridad del campo de la información fue introducida y se perpetúa a través de los diversos orígenes profesionales de las personas en las que se reflejan estos problemas.

(2) Cuando reclama una jurisdicción, una profesión exige de la sociedad que reconozca su estructura cognitiva a través de derechos exclusivos; la jurisdicción no es una cultura, sino una estructura social. Estos derechos exclusivos pueden incluir el monopolio de la práctica y de los pagos públicos, los derechos de disciplina y de empleo, el control del entrenamiento profesional, del reclutamiento y del licenciamiento, por mencionar solo algunos (Abott, 1998, p. 59).

(3) Este decreto informa de que los Consejos de Biblioteconomía tienen como finalidad "orientar, organizar y supervisar la reglamentación del ejercicio de la profesión de bibliotecario y de contribuir al desarrollo profesional".

\section{Referencias}

Abbott, A. (1988). The system of professions: an essay on the division of expert labour. Chicago: The University of Chicago Press, 1988.

Arévalo, J. A.; Vázquez Vázquez, M. (2000). Características del comportamiento del mercado de trabajo en biblioteconomía, archivística y documentación, bienio 98/99. // Anales de Documentación. 3 (2000) 9-24.

Scire. 12 : 2 (jul.-dic. 2006) 27-36. ISSN 1135-3716. 
Bardin, L. (2004). Análise de conteúdo. Lisboa: Edições 70, 2004.

Brasil. Decreto 56726 de 16 ago. 1965. Regulamenta a Lei 4084 de 30 jun. 1962, que dispõe sobre o exercício da profissão do bibliotecário.

Chen, C.-C.; Raskin, S.; Tebbets, D. R. (1984). Products of graduate library and information science schools: unadapted resources? // Education for Information. 2:3 (Sept. 1984) 163-190.

Cronin, B. (1998). Information professionals in the digital age. // The International Information and Library Review, 30:1 (mar. 1998) 37-50.

Cronin, B.; Stiffler, M.; Day, D. (1993). The emergent market for information professionals: educational opportunities and implications. // Library Trends. 42:2 (Fall 1993) 257-276.

Cunha, M. V. (2002). Le profil des professionnels de l'information dans l'État de Santa Catarina, Brésil. // Recherches récentes en sciences de l'information: convergences et dynamiques. Coloque MICS-LERASS (Toulouse, 2002). París: ADBS, 2002. 281-298.

Cunha, M. V. (1998). L'émergence des nouveaux professionnels de l'information: fonctions, compétences, marché. Étude comparée des situations brésilienne et française. Tese (doutorado em Informação Científica e Técnica). París: Conservatoire National des Arts et Métiers, 1998.

Cunha, M. V.; Crivellari, H. M. T. (2006). Paradojas de las profesiones de la información en la sociedad del conocimiento. // Scire: Representación y Organización del Conocimiento. 12:2 (jul.-dic. 2006). 13-26.

Danner, R. A. (1998). Redefining a profession. // Law Library Journal. 90:3 (1998) 315-356.

Dowbor (2001). O que acontece com o trabalho. São Paulo: SENAC, 2001.

García Marco, F. J.; Agustín Lacruz, M. C. A. (2000). Educating the information professional of the $21^{\text {st }}$ century: a ten-point proposal based on the Spanish context. // Education for Information. 18 (2000) 141-153.

Marchiori, P. (1996). Que profissional queremos formar para o século XXI. // Informação \& Informação. 1:1 (1996) 27-34.

Montalli, K. (1997). Perfil do profissional da informação tecnológico e empresarial. // Ciência da Informação. 26:3 (1997) 290-295.

Moore, N. (1987). The emerging markets for librarians and information workers. Boston Spa: The British Library, 1987. (Library and information research report 56)

Moore, N. (1999). Partners in the information society. // Library Record. 101:12 (Dec. 1999). http: //www.la-hq.org.uk/directory/record (2005-06-01).

Mueller, S. P. M. (2004). Uma profissão em evolução: profissionais da informação no Brasil sob a ótica de Abbott. Proposta de estudo. // Mueller, S. P. M; Baptista, S. G. Profissional da informação: o espaço de trabalho. Brasília: Thesaurus, 2004. 23-54.

Saracevic, T. (1995). The interdisciplinary nature of information science. // Ciência da Informação. 24:1 (jan.-abr. 1995) 36-41.

Smit, J. W. (1994). Eu, bibliotecário, rg xxx e cpf yyy, trabalho em arquivo ou museu... algum problema? // Revista Palavra-Chave, 8 (1994) 12-13.

Tarapanoff, K. (1997). Perfil do profissional da informação no Brasil. Brasília: IEL, 1997.

Tomael, M. I.; Alvarenga, G. M. (2000). Profissional da informação: espaço de atuação em empresas industriais. // Perspectivas em Ciência da Informação. 5:1 (2000) 81-90.

Scire. $12: 2$ (jul.-dic. 2006) 27-36. ISSN 1135-3716. 


\section{Apéndice: fuentes}

\section{Listas de discusión}

Lista de discussão da ABECIN. http://www.abecin.org (mayo de 2005).

Lista de discussão do IASI. http://listas.alternex.com.br (mayo de 2004).

Lista de discussão do IBICT. URL: www.ibict.br (mayo de 2005).

\section{Sitios web}

Central de Trabalho e Renda da Confederação Única de Trabalhadores. http://www.central.org (mayo de 2005).

GELRE. Provedor de soluções em recursos humanos. http://www.gelre.com.br (mayo de 2005).

ONG Escola Aprendiz. Site do Aprendiz. http://www.uol.com.br/aprendiz/guiadeemprego (mayo de 2005).

Pontifícia Universidade Católica Do Rio de Janeiro. Serviços. http://www.puc-rio.br/ serviços/empregos.html (mayo de 2005).

Rede Globo. http://empregos.globo.com/parceiros/globo (mayo de 2004).

Infohome. http://www.ofaj.com.br (mayo de 2005).

Bumeran. http://br5.bumeran.com (mayo de 2005). 